P155 (continued)

list users reported higher HEI $(\mathrm{n}=62$, mean $57.8 \pm 11.9$ SD) relative to non-users $(\mathrm{n}=20$, mean $48.0 \pm 11.1 \mathrm{SD}$, $\mathrm{p}=0.002$ ). No difference in mean BMI by use of grocery list was noted $(\mathrm{p}=0.10)$. Covariate-adjusted regression model indicated total HEI was higher among participants who consistently used a grocery list by 7.9 points $(\mathrm{p}=0.02)$, but use of a grocery list was not associated with BMI ( $\mathrm{p}=0.79)$.

Conclusions and Implications: Consistent use of a grocery list was associated with a higher dietary quality, but not BMI. Adopting routine use of grocery lists appears to be a feasible, low-tech, no-cost approach to higher dietary quality.

Funding: NIFA

\section{P156 Web-Based Nutrition Education Program Improves Healthy Eating Strategies in South Asians}

Zubaida Qamar,PhD, zubqam@gmail.com, Texas A\&M University, 3400 Stevenson Boulevard, S-14, Fremont, CA 94538; Alex McIntosh, PhD, Texas A\&M University

Objective: To determine any differences in healthy eating strategies (HES) in South Asians (SA) as a result of an online nutrition education program.

Study Design, Setting, Participants, Intervention: SA have an increased risk of diabetes and cardiovascular diseases in comparison to other ethnic populations in the U.S. To prevent and mitigate the consequences of these conditions, theory-based nutrition programs are needed which can lead to improvement in healthy eating habits. This online nutrition education program was developed following the DESIGN procedure and was guided by the Theory of Planned Behavior. The program focused on consumption of fruits and vegetables, label reading, weight management and physical activity. One of the variables in this project was the measurement of HES. The 15 questions related to HES were adapted from validated survey developed by Norman and colleagues (2010) and scored based on frequency of these strategies. SA participants $(n=166,58.7 \%$ females) completed the pre-test survey, out of which 66 enrolled in the program.

Outcome Measures and Analysis: Statistical analysis included descriptive statistics for demographics, and independent $\mathrm{t}$-tests to determine the differences between preand post-test healthy eating strategies.

Results: Mean age of participants was about 27 years indicating a younger sample. In regards to HES, significant $(\mathrm{p}<0.05)$ differences were observed pre-test (Mean $=47.396$, Standard Deviation $=10.7$ ) and post-test scores $($ Mean $=52.552$, Standard Deviation $=8.97$ ) which suggested an improvement in the healthy eating strategies score after the program.

Conclusions and Implications: Results from this study suggest that a theory-based, online program improved healthy eating strategies score of SA in the
U.S. This can help guide the development of future large scale nutrition education and prevention studies in this population.

Funding: None

\section{P157 Wildland Firefighters' Self-Reported Nutrition and Hydration Concerns that May Impact Health and Safety}

SamanthaWorden, MS, word9534@vandals.uidaho.edu, University of Idaho, 102 East Morton, \#102, Moscow, ID 83843; Callie Collins, University of Idaho; Annie Roe, PhD, RD; Katie Brown, PhD, LDN, RD; Alistair, PhD;

Crystal Kolden, PhD; Andrew Nelson, PhD;

Randy Brooks, PhD; Samantha Ramsay, PhD, LDN, RD

Objective: The purpose of this study was to identify wildland firefighters' (WLFFs) self-reported hydration and nutrition practices, they perceived may impact health and safety while on an active fire assignment in the United States.

Study Design, Setting, Participants: Using an online format, WLFFs $(n=422)$ were asked two open-ended questions and five demographic questions. One question asked participants to indicate nutrition and hydration factors that contribute to common health and safety accidents. The second question asked participants whether they had additional comments related to health and safety.

Outcome Measures and Analysis: Descriptive data was tabulated for the demographic questions, and content analysis of narrative text was used to identify major themes.

Results: WLFFs consisted primarily of firefighter crew members $(\mathrm{n}=302,72 \%)$, and managerial positions $(\mathrm{n}=120,28.1 \%)$. The majority had a college degree or higher $(n=261,61.8 \%)$. Five overarching themes were identified in WLFFs' perceptions of the nutrition and hydration factors that influenced health and safety: practices that cause dehydration (e.g. caffeine intake); challenges and successes with hydration and nutrition; food quality as a priority (e.g. increasing nutrient dense foods and decreasing processed and convenient foods); the impact of sleep on overall diet; and WLFFs' lack of knowledge of healthful nutrition and hydration practices.

Conclusions and Implications: WLFFs reported nutrition and hydration practices that they perceived contribute to health and safety while on a fire assignment. WLFFs responses indicate a greater understanding of nutrition and hydration practices is needed to minimize accidents during fire operations, and to establish nutrition and hydration guidelines for agencies that fight fire.

Funding: None

\section{P158 Young Adult African American Dietary Patterns Vary by Ethnic Identity}

Donna Winham, DPH, RD, dwinham@iastate.edu, Iowa State University, 2302 Osborn Drive, Ames, IA 50011

Objective: The study evaluated associations between dietary intakes and ethnic identity among young adult

Continued on page S86 